\title{
La Unión Europea, el Líbano y los refugiados sirios ${ }^{1}$
}

\author{
The European Union, Lebanon, and the Syrian refugees
}

\author{
Javier Lion Bustillo \\ Universidad Complutense de Madrid \\ flion@ucm.es
}

Sumario: I. Introducción.-II. El impacto de la acogida de refugiados en países cercanos a un conflicto: el caso libanés. - III. La guerra civil siria y la nueva oleada de refugiados.-IV. La respuesta de la UE.-V. Entre la europeización y el statu quo.-VI. Conclusiones.-VII. Bibliografía.

Resumen: El propósito de este artículo consiste en evaluar la coherencia de la acción exterior de la Unión Europea entre sus relaciones con el Líbano y su política hacia los refugiados sirios. Para ello, se llevará a cabo un contraste entre los objetivos de la Política Exterior y de Seguridad Común y los del Espacio de Libertad, Seguridad y Justicia. La primera declara promover la estabilidad en ese país, además de extender hacia él las ideas europeas en materia de democratización, derechos humanos y buen gobierno. El segundo busca que los refugiados permanezcan en países vecinos (como es el caso del Líbano) y que se evite su llegada a Europa. Los resultados de este trabajo subrayan que esta notable presencia de refugiados en un país con recursos limitados, con una administración pública ineficaz y clientelista, y con un sistema político inestable está conduciendo a evidentes tensiones, que se pueden ver reforzadas si buena parte de esos refugiados permanece indefinidamente en el país. De este modo, la obsesión por tratar a los refugiados como una amenaza a su seguridad debilita la coherencia de la acción exterior de la UE y perjudica el logro de sus otros objetivos.

Palabras clave: Líbano, refugiados, Política Exterior y de Seguridad Común, Unión Europea, Espacio de Libertad, Seguridad y Justicia.

Abstract: The purpose of this article consists of assessing the coherence of the European Union external action between its relations with Lebanon and its policy

${ }^{1}$ Este artículo se inscribe en el marco del proyecto de investigación dirigido por Laura Feliú Martínez y Ferrán Izquierdo Brichs, «Dinámicas y actores transnacionales en Oriente Medio y Norte de África (MENA): una genealogía histórica de élites y movimientos sociales entre lo local y lo global» (Ministerio de Economía y Competitividad, Agencia Estatal de Investigación y Fondo Europeo de Desarrollo Regional) (HAR2016-77876-P). 
towards the Syrian refugees. With this objective, I will contrast the objectives of the Common Foreign and Security Policy and those of the Area of Freedom, Security and Justice. The former is aimed at promoting stability in Lebanon and spreading to that country the European views of democracy, human rights and good governance. The latter wants the refugees to remain in neighbouring countries (like Lebanon) and prevent their arrival into Europe. The results of this work highlight that the presence of such a high number of refugees in a country with very limited resources, a clientelist and inefficient public administration, and an unstable political system is leading to open tensions that will grow if those refugees remain in Lebanon indefinitely. Therefore, the obsession for treating the refugees as a security threat is weakening the coherence of the EU external action and undermining the achievement of other objectives.

Keywords: Lebanon, refugees, Common Foreign and Security Policy, European Union, Area of Freedom, Security and Justice.

\section{Introducción}

El Líbano es un país en el que la fractura generada por la guerra civil (1975-1990) sigue estando presente en muchos aspectos de la realidad. En aquella crisis, un factor de gran importancia fue la presencia de un elevado número de refugiados palestinos, algunos de los cuales empleaban la zona sur como plataforma en su lucha contra Israel. Además, fuerzas palestinas pasaron a tomar parte en los enfrentamientos armados entre diferentes milicias libanesas. Tras los Acuerdos de Ta'if (1989), el país entró en una fase de pacificación bajo hegemonía siria, en tanto que Israel controlaba su parte meridional. Pero la retirada israelí en 2000 y la de Siria en 2005 reabrieron la pugna por el control del sistema político entre las distintas facciones, y si bien la paz se ha mantenido desde entonces, no han faltado incidentes armados que han hecho pensar en la posibilidad de una nueva guerra civil. La llegada de un elevado número de refugiados sirios desde 2011 provocó el que resurgieran los temores a un retorno al pasado, especialmente cuando se percibió que algunas milicias sirias utilizaban el territorio libanés como base para sus operaciones, en las que contaron con el apoyo de grupos locales. En definitiva, el Líbano corrió un serio riesgo de experimentar un desbordamiento del conflicto sirio, poniendo en evidencia su fragilidad ante estas situaciones ${ }^{2}$.

2 Javier Lion Bustillo, «Líbano y Siria: entre la disociación y el desbordamiento», Revista CIDOB d'Afers Internacionals, n. ${ }^{\circ} 108$ (2014): 213-235. 
La UE ha llevado a cabo una política de gestión del éxodo de refugiados procedentes de Siria predominantemente basada en que el mismo constituía un desafío a su propia seguridad y en que la mejor respuesta consistía en externalizar el problema, de manera que los refugiados permanecieran en países vecinos como el Líbano, a pesar de sus limitadas capacidades en el área de las políticas públicas. Pero sobre todo el problema planteado fue de carácter político, ya que la presencia de los refugiados podía alterar allí los frágiles equilibrios demográficos, confesionales y políticos existentes, colocando al país ante un nuevo reto para su estabilidad. No obstante, esta política europea contenía un riesgo para sí misma, ya que cualquier estallido de violencia en el Líbano no haría sino agravar la propia crisis de refugiados en dirección a Europa. Todo esto nos conduce a tratar de evaluar la coherencia entre la política de la UE hacia el Líbano y sus iniciativas hacia los refugiados sirios.

La estructura de este trabajo comenzará con la exposición de las aproximaciones teóricas a los posibles efectos de la llegada de gran número de refugiados a países vecinos de aquellos envueltos en conflictos armados. También se explicarán las especificidades del Estado libanés y su vulnerabilidad hacia las crisis de refugiados que alteren los equilibrios internos de poder. En segundo lugar, se repasará el proceso de llegada y asentamiento de los refugiados sirios en el Líbano desde el inicio de la guerra civil, así como su efecto en la política libanesa. A continuación, se analizarán las políticas de la UE hacia el Líbano y hacia los refugiados sirios. En el siguiente apartado, se evalúa hasta qué punto existe una coherencia entre los diferentes objetivos de las políticas de la UE en el Líbano repasando sus efectos prácticos. Finalmente, se extraerán algunas conclusiones que evidencian esa falta de coherencia antes señalada.

\section{El impacto de la acogida de refugiados en países cercanos a un conflicto. El caso libanés}

La proliferación de oleadas de refugiados en las últimas décadas ha fomentado los estudios sobre sus posibles efectos. Para Myron Weiner (1996), existe la tendencia a que ciertos espacios geográficos se conviertan en lo que denomina «bad neighborhoods», es decir, «regions with a number of countries in which violence and brutality impel large numbers of people to cross international borders in search of security» ${ }^{3}$.

3 Myron Weiner. «Bad Neighbors, Bad Neighborhoods: An Inquiry into the Causes of Refugee Flows», International Security, n. . 21, 1 (1996): 26-28. 
En estos contextos, es probable que una guerra civil en un país acabe afectando a sus vecinos, dado que entre los refugiados se pueden infiltrar algunos combatientes que desean utilizar el territorio del Estado de acogida como base para nuevos ataques en su país de origen, convirtiéndose en lo que se ha denominado «refugiados-combatientes» ${ }^{4}$. Por otra parte, las autoridades del país de acogida pueden bien respaldar esta actitud o bien oponerse a ella por diferentes motivos, que van desde un respeto escrupuloso a su neutralidad y soberanía, pasando por el temor a que esa situación conduzca a incidentes con las autoridades del país vecino o a que los combates se extiendan a su propio territorio. El mayor peligro es que los combatientes foráneos lleguen a convertirse en un poder de facto en el país de acogida, colocando zonas de su territorio bajo su propio control y desafiando a sus autoridades. Esta situación sería especialmente inflamable en el caso de países en los que existe un gran fraccionamiento de su cultura política, con comunidades fuertemente vinculadas con alguno o varios de los grupos enfrentados en la guerra civil vecina, con el consiguiente riesgo de desbordamiento de dicho conflicto ${ }^{5}$. Por el contrario, otros autores subrayan que esa militarización de los refugiados constituye una anomalía y que el intento de aplicar un paradigma de seguridad a ese colectivo estaría vinculado al desarrollo de unas políticas cada vez más restrictivas por parte de los países occidentales ${ }^{6}$.

En el caso específico del Líbano, su sistema político se caracterizó desde sus inicios por un modelo consociacional, que implicó la decisión de compartir el poder entre los diferentes grupos confesionales de tal modo que las principales magistraturas políticas del país, así como las principales posiciones en la administración pública, quedaron repartidas entre ellos. En realidad, el aparato del Estado se convirtió en un complemento de las propias estructuras clientelistas del sector privado, de manera que ambas dimensiones permitieron un liderazgo estable dentro de cada comunidad confesional en las dimensiones política y social a cargo de las mismas personas, los denominados zuama. Sin embargo, este modelo ha sido históricamente incapaz de mantener el control sobre la violencia interconfesional, que creció debido a factores como los cambios demográficos, la lucha por el territorio o el impacto de las

${ }^{4}$ Aristide Zolberg, Astri Suhrke y Sergio Aguayo, Escape from Violence: Conflict and the Refugee Crisis in the Developing World (Nueva York: Oxford University Press, 1989), 275. Sarah K. Lischer, «Catalysts of Conflict: How Refugee Crises Lead to the Spread of Civil War» (Tesis Doctoral, Massachusetts Institute of Technology, 2002), 10, https://core. ac.uk/display/4393676

${ }^{5}$ Lischer, «Catalysts...», 23. David Carment, Patrick James y Zeynep Taydas, «The Internationalization of Ethnic Conflict: State, Society, and Synthesis», International Studies Review, n. ${ }^{\circ} 11,1$ (2009): 66-68.

${ }^{6}$ Reynoud Leenders, «Refugee Warriors or War-Refugees? Iraqi Refugees` Predicament in Syria, Jordan and Lebanon». Mediterranean Politics, n. ${ }^{\circ}$ 14, 3(2009): 357. 
crisis regionales, desembocando en la guerra civil de 1975-907. Por su parte, hay autores que consideran que la debilidad del Líbano no procede de su sistema político, sino de su carácter de Estado de reciente creación, lo que le haría vulnerable dada su ausencia de legitimidad y trataría de ser compensado por los diferentes partidos a través de alianzas en el sistema internacional. En tales circunstancias, las tensiones en dicho sistema desembocarían fácilmente en estallidos de violencia e incluso en guerras civiles ${ }^{8}$.

La guerra civil libanesa (1975-90) constituyó un buen ejemplo de esta dinámica, ya que el enorme fraccionamiento de la sociedad y la clase política se mezcló con un escenario de gran inestabilidad regional, de manera que cada facción buscó respaldo en el exterior para garantizar su poder. De hecho, entre sus causas podemos destacar los cambios demográficos y la consiguiente ruptura de los equilibrios entre los diferentes grupos confesionales. En ese contexto, los refugiados palestinos decidieron implicarse en el conflicto interno para garantizar su supervivencia como actor autónomo al margen del control del Estado libanés o de otros gobiernos árabes, lo que acentuó más la ruptura del tradicional consenso que sostenía el sistema institucional. Esto ha conducido a muchos libaneses a considerar la guerra civil principalmente como el resultado de las acciones de esos refugiados, una opinión fomentada por diversos partidos 9 .

Tras el final de la guerra civil, Siria se convirtió en la potencia hegemónica en el Líbano mediante la presencia de sus tropas y de sus servicios de seguridad. Sin embargo, esa posición se fue deteriorando tras la invasión estadounidense de Irak (2003), ya que Washington decidió castigar a Damasco por su oposición a la misma. Ante este giro, mientras unos partidos prefirieron conservar el apoyo sirio, otros pasaron a la oposición. La oleada de protestas en las calles tras el asesinato del exPrimer Ministro Rafik Hariri (2005) forzó la salida de las tropas sirias, pero a partir de ese momento las rivalidades entre los partidos desembocaron en la división del país en dos coaliciones enfrentadas, el 8 y el 14 Marzo: la primera se alineó con Irán y Siria, y la segunda con Arabia Saudí y Estados Unidos ${ }^{10}$.

7 Tamirace Fakhoury, Democracy and Power-Sharing in Stormy Weather: The Case of Lebanon (Wiesbaden: Springer, 2009), 77-92. Samir Khalaf, Civil and Uncivil Violence in Lebanon (Nueva York: Columbia University Press, 2002), 43-48.

8 Adham Saouli, «Stability under Late State Formation: The Case of Lebanon». Cambridge Review of International Affairs, n. ${ }^{\circ}$ 19, 4 (2006): 701-717.

${ }^{9}$ Khalaf, Civil and Uncivil..., 224.

${ }^{10}$ La Coalición 8 de Marzo está integrada por los dos grandes partidos chiíes, Hezbollah y Amal. Además, cuenta con la colaboración del principal partido cristiano, el Movimiento Patriótico Libre del actual presidente, Michel Aoun. Por su parte, el 14 de Marzo incluye al principal partido suní, el Movimiento de Futuro de la familia Hariri, así como a los partidos cristianos de las Fuerzas Libanesas y la Falange. 
En este clima de división interna, la única fórmula que ha permitido un cierto nivel de estabilidad y calma ha sido el recurso a gobiernos de unidad nacional en los que están representadas ambas coaliciones rivales, de manera que el modelo consociacional se ha visto reforzado. El problema es que la toma de decisiones se ha hecho enormemente compleja y su efecto ha sido el eludir aquellas que puedan suscitar más controversia y que afecten a los intereses de los dirigentes políticos o sus allegados (subidas de impuestos, cambios en el mercado laboral, desarrollo de una administración pública neutral y eficiente, desarme de todos los grupos, etc.). Por lo tanto, los principales problemas del país (corrupción, clientelismo, altísima deuda pública, déficits en los servicios públicos...) no han encontrado una solución viable. En este contexto, los políticos libaneses de todas las tendencias han recurrido de manera sistemática a solicitar dinero de los diferentes actores internacionales con el argumento de que el país estaba al borde del hundimiento y de que era posible una nueva guerra civil ${ }^{11}$.

\section{La guerra civil siria y la nueva oleada de refugiados}

Con el comienzo de la guerra civil en Siria (julio de 2011), los primeros desplazados por el conflicto llegaron a la frontera libanesa, que pudieron atravesar sin problemas ya que las autoridades de Beirut optaron por una política de puertas abiertas por distintos factores, destacando los lazos históricos, sociales y culturales entre ambos países. De hecho, se trataba de un reducido número refugiados procedentes principalmente del área de Homs, que se instalaron en viviendas de familiares y amigos ${ }^{12}$. Otro factor relevante fue que los distintos partidos veían los acontecimientos en Siria desde una perspectiva opuesta, por lo que resultaba muy difícil el articular una política coherente y lo que predominó así fue la inacción ${ }^{13}$. Poco a poco, el

${ }^{11}$ Nisreen Salti y Jad Chaaban, «The Role of Sectarianism in the Allocation of Public Expenditure in Postwar Lebanon», International Journal of Middle East Studies, n. ${ }^{\circ} 42,4$ (2010): 651-652. Mounir Mahmalat y Sami Atallah «Why Does Lebanon Need CEDRE? How Fiscal Mismanagement and Low Taxation on Wealth Necessitate International Assistance», The Lebanese Center Policy Studies, Policy Brief n. ${ }^{\circ} 34$ (2018), 4, acceso el 5 de abril de 2020, https://www.lcps-lebanon.org/publication.php?id=318

12 Ninette Kelley, «Responding to a Refugee Influx: Lessons from Lebanon». Journal on Migration and Human Security, n. ${ }^{\circ}$ 5, 1 (2017): 85.

${ }^{13}$ El Gobierno dirigido por Najib Mikati contaba entonces solamente con el apoyo del 8 de Marzo. Hala Naufal, «Syrian Refugees in Lebanon: The Humanitarian Approach under Political Divisions», Robert Schumman Centre for Advanced Studies, San Domenico de Fiesole, European University Institute, 2012-13, 9-12, acceso el 24 de marzo de 2020, https:// cadmus.eui.eu/handle/1814/24835 
número de refugiados fue creciendo hasta situarse en un millón en 2014, lo que forzó la creación de una célula de crisis entre diversos ministerios para afrontar la situación, al tiempo que entre responsables gubernamentales se expresaba la incapacidad del país para hacer frente a la situación y se exigía a la comunidad internacional el que asumiera sus responsabilidades en la gestión del problema. Ante el precedente palestino, las autoridades no permitieron la creación de campos, aunque en la práctica éstos surgieron con carácter informal, mientras que otras veces los campos palestinos ya existentes se convirtieron en el destino de esos refugiados ${ }^{14}$.

Dados los estrechos lazos históricos entre ambos países, resultaba inevitable que los efectos del conflicto dejaran su huella en una arena política libanesa ya de por sí enormemente fracturada, con largas etapas de gobiernos provisionales. La Coalición 8 de Marzo mostró su cercanía al régimen de Damasco, mientras que el 14 de Marzo se inclinaba por apoyar a los grupos insurgentes. Así, Herzbollah fue implicándose crecientemente en la guerra en Siria mediante el envío de un número notable de combatientes, en tanto que el Movimiento de Futuro ayudaba a aquellos grupos (especialmente milicias salafistas libanesas) que deseaban atravesar la frontera para combatir contra Bashar al-Asad. De este modo, los espacios fronterizos entre ambos países se convirtieron en un territorio cuyo control resultaba clave para el sostenimiento de las fuerzas insurgentes sirias dada su importancia logística y su uso como plataforma en la que refugiarse o desde la que atacar a las tropas del régimen. Por su parte, Hezbollah trataba de impedir esto, colaborando con el Ejército sirio, mientras que el Ejército libanés y sus fuerzas de seguridad se hallaban sobrepasadas en el dominio de una región como el Valle de la Bekaa, en la que la complejidad orográfica dificulta esa tarea ${ }^{15}$.

Los políticos libaneses trataron de consensuar una política con respecto a Siria, reflejada en el denominado Acuerdo de Baabda (junio de 2012), que implicaba la neutralidad del país hacia el conflicto. Pero lo cierto es que ambas coaliciones siguieron apoyando a sus respectivos aliados al otro lado de la frontera, lo que tensó enormemente las relaciones entre las distintas comunidades (especialmente entre suníes por un lado y chiíes y alauíes por

${ }^{14}$ Naufal, «Syrian Refugees...», 13. Filippo Dionigi, «The Syrian Refugee Crisis in Lebanon: State Fragility and Social Resilience». LSE Middle East Center Paper Series, n. ${ }^{\circ} 15$ (2014), 9-13, acceso el 4 de marzo de 2020, http://eprints.lse.ac.uk/65565/1/Dionigi_Syrian_ Refugees\%20in\%20Lebanon_Author_2016.pdf

15 International Crisis Group, «Too Close for Comfort: Syrians in Lebanon», Report $n .^{o}$ 141, Beirut/Bruselas, 2012, 1-3, acceso el 2 de abril de 2020, https://www.crisisgroup. org/middle-east-north-africa/eastern-mediterranean/lebanon/too-close-comfort-syrianslebanon\#: :text=As\%20the $\% 20$ Syrian $\% 20$ conflict $\% 20$ increasingly,volatile $\% 20$ region $\% 20$ can\%20ill\%20afford. 
otro) y entre los partidos políticos. De hecho, esta rivalidad fue adoptando a menudo un tono cada vez más sectario y confesional. Por otra parte, los refugiados no estaban dispersos en el conjunto del país, sino que se hallaban fuertemente concentrados en algunas zonas como la Bekaa Occidental, Zahle, Baalbek-Hermel, Akkar, Baabda o Trípoli. Su presencia suscitó las primeras reacciones negativas entre algunos políticos libaneses, especialmente entre los cristianos maronitas, como el ministro de Exteriores Gebran Bassil o el líder falangista Sami Gemayel, quienes apoyaban la idea de crear campos para acogerlos, pero situados en la zona fronteriza con Siria y no en el interior del país ${ }^{16}$.

Las diferentes preferencias políticas de los refugiados influían también en el tratamiento que las distintas fuerzas políticas estaban dispuestas a otorgarles ${ }^{17}$. Así, la presencia mayoritaria de suníes, muchos de los cuales eran opuestos al régimen de Damasco, desató las críticas de los partidos del 8 de Marzo, que temían el que algunos de ellos pudieran convertirse tanto en participantes en la guerra civil siria como en combatientes en los enfrentamientos violentos entre milicias libanesas. Los escenarios más preocupantes en este sentido eran tanto el Valle de la Bekaa como la ciudad de Trípoli.

En la Bekaa, buena parte del territorio estaba bajo el control de Hezbollah, por lo que resultaba complejo el crear una estructura logística que apoyara las actividades de los insurgentes sirios. Sin embargo, en algunos espacios (como la villa de Arsal) el predomino del Movimiento de Futuro y de otras organizaciones suníes generaba un escenario propicio, lo que motivó que tanto el Ejército libanés como Hezbollah desarrollaran una labor tendente a desmantelar cualquier infraestructura de los insurgentes sirios, dándose unas relaciones especialmente tensas con la población local. El punto culminante tuvo lugar en 2013, cuando la situación en el escenario bélico sirio parecía favorecer a las fuerzas opositoras, que trataban de arrebatar al gobierno de Damasco el control sobre la autopista M-5, de gran importancia económica y estratégica. Su proximidad a la frontera libanesa hizo que algunas organizaciones opositoras (especialmente grupos yihadistas) trataran de emplear el territorio libanés en sus operaciones ${ }^{18}$.

En el caso específico de la ciudad de Arsal, ésta se caracteriza por unos estrechos vínculos económico-sociales con la región siria de Homs. Al mismo tiempo, se trata de una zona tradicionalmente olvidada por las autoridades de Beirut, ya que al ser una población mayoritariamente suní en

16 «Gemayel Sounds Alarm about Refugee Crisis», Now, 15 de abril de 2013, acceso el 27 de marzo de 2020, http://now.mmedia.me/lb/en/archive/gemayel-sounds-alarm-aboutrefugee-crisis

17 Dionigi, «The Syrian Refugee...», 14.

18 Javier Lion Bustillo, «Líbano y Siria...», 223. 
medio de una región dominada por Hezbollah, no es electoralmente útil ni para ese partido ni para el Movimiento de Futuro, por lo que el acceso a los servicios sociales es muy precario. Su estructura económica es arcaica, lo que ha provocado que el fenómeno del contrabando haya tenido un peso notable ${ }^{19}$. La amarga experiencia de la ocupación siria desde la guerra civil hasta 2005 condujo a que sus habitantes apoyaran la insurrección contra Asad y acogieran a numerosos refugiados. Sin embargo, la situación se hizo mucho más compleja cuando el número de éstos cuadruplicó el de residentes libaneses, provocando subidas de precios, mayor competencia por el empleo y problemas de vivienda, de acceso al agua y a la electricidad. Pero el principal factor desestabilizador lo constituyó la creciente presencia de combatientes opositores sirios, quienes aprovechaban la frontera para sus fines militares. La toma de la ciudad de Qusair (junio de 2013) por las tropas de Damasco y de Hezbollah, y su ofensiva contra los insurgentes llevó a éstos a replegarse hacia la frontera y a aumentar su presencia en Arsal, implicándose crecientemente en la política libanesa. Estos insurgentes pertenecían mayoritariamente a grupos yihadistas (Jabhat al-Nusra y el Estado Islámico) que decidieron convertir Arsal en un feudo bajo su control, al tiempo que perpetraban atentados contra Hezbollah y el Ejército libanés. Estos provocaron una elevación de las tensiones en el país entre las coaliciones 8 y 14 de Marzo, colocándolo al borde del enfrentamiento armado, con una sucesión de asesinatos de personalidades y atentados indiscriminados. Esta tensión sólo se fue difuminando a medida que el Ejército optó por una actuación más decidida en su lucha contra el yihadismo en Arsal, con enfrentamientos directos con los grupos allí establecidos que dejaron numerosos muertos y un elevado nivel de destrucción en la ciudad hasta que los yihadistas la abandonaron (agosto de 2014). No obstante, esta acción fue tachada de desproporcionada entre una parte relevante de la población suní, que consideraba que el Ejército estaba cooperando con Hezbollah en su lucha contra los insurgentes sirios ${ }^{20}$. De hecho, sólo una nueva ofensiva en verano de 2017 acabó con la presencia yihadista.

Otra posibilidad fue la de que algunos refugiados fueran reclutados para las luchas puramente intralibanesas que de vez en cuando sacuden algunas áreas del país. Un claro ejemplo es la ciudad costera de Trípoli, tremendamente empobrecida en las últimas décadas debido a factores como la guerra civil, la asfixiante presencia de las tropas sirias hasta 2005, las tensiones

${ }^{19}$ International Crisis Group, «Arsal in the Crosshairs: The Predicament of a Small Lebanese Border Town», Middle East Briefing $n .^{\circ} 46$, Beirut/Bruselas, 4-5, acceso el 5 de marzo de 2020, https://static1.squarespace.com/static/599720dc59cc68c3683049bc/t/5d64233 3b31a9d0001353837/1566843701639/Tripoli+RIT+Case+Study+Report.pdf

${ }^{20}$ International Crisis Group, «Arsal...», 8-14. 
existentes entre el barrio suní de Bab al-Tabbaneh y el alauí de Jabal Mohsen, el olvido de las élites económicas y políticas, un sistema educativo ineficaz o los elevados índices de desempleo. A partir de 2012 se registró la llegada de un elevado número de refugiados procedentes de Siria, algunos de los cuales eran palestinos establecidos en el país vecino ${ }^{21}$. De hecho, muchos de los refugiados se han asentado en los campos palestinos existentes en Trípoli, además de en pequeños campos informales. La cifra global de refugiados alcanzó más de 70.000, lo que provocó una sobrecarga en los servicios públicos para los ciudadanos libaneses y para los refugiados palestinos asentados en el pasado. Igualmente, creció rápidamente la demanda de empleo, lo que supuso una caída de los salarios. En definitiva, todo esto fomentó el descontento entre la población, que generalmente culpó a las autoridades de Beirut por el olvido en que mantenían a la ciudad ${ }^{22}$.

La presencia en Trípoli de grupos yihadistas locales y palestinos ofrecía la posibilidad para sus correligionarios sirios de desarrollar una labor de cooperación en su lucha, integrándose en la arena política libanesa. Por ejemplo, algunos de ellos se implicaron en los tiroteos entre Bab al-Tabbaneh y Jabal Mohsen, aprovechándose del componente confesional del combate contra los alauíes. A raíz de esto, la situación en Trípoli se hizo especialmente inflamable en el período de 2013-2015, sucediéndose atentados indiscriminados que provocaron un gran número de víctimas. Nuevamente, fue el Ejército quien debió intervenir para desarticular el foco yihadista, lo que generó entre muchos suníes la sensación de una progresiva marginación de su comunidad ${ }^{23}$.

Acontecimientos como los de Arsal y Trípoli provocaron un intenso debate entre la clase política libanesa en torno a la cuestión de si el país debía seguir recibiendo refugiados sirios y en qué número, lanzado principalmente por los partidos cristianos. El entonces ministro de Exteriores Gebran Bassil, del Movimiento Patriótico Libre, descartó la posibilidad de una instalación permanente de los refugiados sirios en el Líbano (que consideró inconstitucional) y propuso la necesidad de adoptar mayores exigencias con respecto a la llegada de nuevos refugiados. Bassil recalcó en sus

${ }^{21}$ Más de 30.000 palestinos procedentes de Siria se hallaban registrados oficialmente en el Líbano en 2018, pero se considera que su número es más elevado, ya que su registro oficial es más complejo y costoso que para los refugiados sirios.

${ }^{22}$ Khaled Ismail, Claire Wilson y Nathan Cohen-Fournier, «Syrian Refugees in Tripoli, Lebanon», Feinstein International Center, 2017, acceso el 6 de marzo de 2020, https://static1.squarespace.com/static/599720dc59cc68c3683049bc/t/5d642333b31 a9d0001353837/1566843701639/Tripoli+RIT+Case+Study+Report.pdf

${ }^{23}$ Michael Young, «In Tripoli, a Conflict Exploited by All», The Daily Star, 8 de diciembre de 2013, acceso el 8 de diciembre de 2013, http://www.daylystar.com.lb/Opinion/ Columnist/2013/Dec-05/239935-in-tripoli-a-conflict-exploited-by-all.ashx ₹axzz2vSdCbw27 
intervenciones que el Líbano no aceptaría las presiones internacionales en el tema de los refugiados y que el país era soberano en ese terreno, aunque al mismo tiempo subrayó que cumpliría con sus deberes humanitarios. Pero destacó que la comunidad internacional había sido incapaz de aportar a la sociedad libanesa la ayuda necesaria en este aspecto, de modo que «They have restricted the aid to Syrian refugees and not the Lebanese» ${ }^{24}$.

\section{La respuesta de la $\mathrm{UE}$}

La Política Exterior y de Seguridad Común (PESC) de la UE hacia los vecinos mediterráneos (y hacia el Líbano en particular) ha experimentado ciertos cambios sutiles pero relevantes en cuanto a sus objetivos. Si originalmente se hablaba de fomentar la democratización, el respeto a los derechos humanos y el Estado de Derecho ${ }^{25}$, en los últimos años el principal énfasis se ha otorgado a la idea de estabilidad, lo que cuestiona su discurso reformista. La UE promueve en el Oriente Medio regímenes que mantengan una política favorable a los intereses occidentales, tales como la apertura y liberalización de sus mercados, la resolución pacífica del conflicto árabe-israelí, la lucha contra el terrorismo yihadista, el control de las migraciones irregulares o el alineamiento geopolítico con Occidente ${ }^{26}$. En este sentido, trata de minimizar la influencia de actores como Siria o Irán (y de sus aliados regionales, a los que considera como rivales en la región). No obstante, el Líbano es un país en el que la influencia de ambos Estados es muy notable a través de la Coalición 8 de Marzo, que ha mantenido en los últimos años una fuerte presencia en el Gobierno de Beirut. Pero si bien la UE desearía ver disminuida esa presencia en favor de sus rivales del 14 de Marzo, no comparte la visión que a veces se ha sostenido desde Washington o Tel Aviv de utilizar la fuerza para imponer un cambio en los equili-

${ }^{24}$ Hassan Lakkis, «Bassil: Lebanon, not UN, Sets Refugee Policies», The Daily Star, 11 de noviembre de 2014, acceso el 6 de junio de 2020, https://www.dailystar.com.lb/News/ Lebanon-News/2014/Nov-11/277234-bassil-lebanon-not-un-sets-refugee-policies.ashx

25 European Union, «Lebanon. Country Strategy Paper 2007-13», 2007, 3-5, acceso el 5 de noviembre de 2019, https://library.euneighbours.eu/content/lebanon-country-strategypaper-2007-2013-and-national-indicative-programme-2007-2010

${ }_{26}$ Tamirace Fakhoury, «The European Union's Engagement in Conflict Processes and Conflict Spillovers: The Case of Lebanon Since the Onset of the Syrian War», European Foreign Affairs Review, n. ${ }^{\circ} 22,1$ (2017): 39-58. European Commission, «Programming of the European Neighbourhood Instrument (ENI) (20142020) - Single Support Framework for EU support to Lebanon (2017-2020)», acceso el 12 de noviembre de 2019, https://ec.europa.eu/ neighbourhoodenlargement/sites/near/files/single_support_framework_for_eu_support_to_ lebanon_20172020_annex.pdf (25.01.2019) 
brios de poder en el Líbano. Esto podría provocar allí una guerra civil que tendría desastrosas consecuencias sobre la propia UE al generar presumiblemente una gran oleada de refugiados. En otras palabras, la retórica democratizadora encuentra sus límites en un entorno en el que prevalece una política basada en intereses confesionales y donde el pluralismo existente no es útil para crear un Estado más fuerte y eficaz ${ }^{27}$.

Por lo tanto, la política de la UE hacia el Líbano se basa en un equilibrio muy sutil entre diferentes objetivos. Por un lado, desea mantener una cierta estabilidad política, si bien la misma debe ser compatible con un debilitamiento del poder de la Coalición 8 de Marzo (especialmente de Hezbollah) en favor de sus rivales del 14 de Marzo. Pero esto no debe de ir en detrimento de la democratización del país y del respeto a los derechos humanos, de acuerdo con una visión normativa. La UE estableció en 2006 un Acuerdo de Asociación con el Líbano con el objetivo de reforzar la posición del nuevo Gobierno de Beirut establecido tras la revuelta de 2005 y controlado por la Coalición 14 de Marzo. Sin embargo, la división política dio al traste con estos planes y desembocó en gobiernos de amplia mayoría respaldados por partidos de ambas coaliciones que han frenado las reformas y se han limitado a tratar de evitar un nuevo conflicto civil. Esto ha provocado que la UE careciera de una influencia significativa en el país.

Por su parte, la política europea dentro del Espacio de Libertad, Seguridad y Justicia (ELSJ) se ocupa de la gestión de los refugiados y demandantes de asilo, área en la que ha predominado el principio de externalización, según el cual los refugiados deben encontrar acomodo en los países más próxi$\operatorname{mos}^{28}$. Esta posición se explica por las reticencias europeas hacia las migraciones, consideradas a menudo como una amenaza. En este sentido, dado que muchos refugiados llegan a Europa a través de vías no legales, ha habido una tendencia a mezclar la lucha contra la inmigración irregular con el problema de los refugiados, de modo que la externalización se convierte en un mecanismo de seguridad que evita su acogida. Sin embargo, esta política puede chocar con otros objetivos de la acción exterior de la UE, así como suscitar

${ }^{27}$ Fakhoury, «The European Union's...», 54. Nathalie Tocci, «What Went Wrong? The Impact of Western Policies towards Hamas and Hizbollah», CEPS Policy Brief $n .^{\circ} 135,2007$, acceso el 19 de marzo de 2020, https://core.ac.uk/download/pdf/5080237.pdf. Peter Seeberg, «The EU as a Realist Actor in Normative Clothes: EU Democracy Promotion in Lebanon and the European Neighbourhood Policy», Democratization, n. ${ }^{\circ}$ 16, 1 (2008): 81-99.

28 Por externalización de los controles migratorios se entiende «...extraterritorial state actions to prevent migrants, including asylum seekers, from entering the legal jurisdictions or territories of destination countries or regions or making them legally inadmissible without individually considering the merits of their protection claims». Bill Frelick, Ian Kysel y Jennifer Podkul, «The Impact of Externalization of Migration Controls on the Rights of Asylum Seekers and Other Migrant», Journal on Migration and Human Security, n. ${ }^{\circ} 4,4$ (2016): 193. 
críticas hacia esa externalización si los países vecinos eluden la aplicación del principio de no repatriación forzosa a sus países de origen, si la seguridad de los refugiados está en peligro, o si sus derechos humanos son violados de modo flagrante ${ }^{29}$. En otras palabras, hay una contradicción entre el interés de algunos políticos europeos en impedir la llegada de un número significativo de refugiados y las propias normas y principios de la $\mathrm{UE}^{30}$.

Desde el inicio de la crisis siria, la UE ha practicado una política tendente a externalizar la gestión del éxodo de refugiados hacia sus vecinos del Oriente Medio. Dada la completa oposición de algunos países (Israel, Arabia Saudí, Emiratos Árabes Unidos...) a aceptar esos refugiados, la gran mayoría de ellos se instaló en Turquía, Líbano y Jordania ${ }^{31}$. En el caso libanés, la UE ha exigido a las autoridades de Beirut tanto el aceptar la entrada de los refugiados procedentes de Siria como su permanencia en el país mientras falten las condiciones necesarias para su retorno, subrayando además el impacto económico positivo que esos refugiados pueden tener gracias a su formación y a un aumento de la demanda ${ }^{32}$. Pero el impulso decisivo a esta política se dio con la crisis provocada por la llegada a la UE de un elevado número de solicitantes de asilo en el período 2014-15. Ante la presión existente sobre países europeos como Italia y Grecia, la UE aprobó un mecanismo de reubicación temporal de solicitantes de asilo, si bien el mismo no pudo ser luego aplicado por las resistencias de algunos socios. Sin embargo, en ningún caso se manejó la posibilidad de un sistema similar para aligerar los problemas de los países vecinos. Por el contrario, simplemente se optó por darles un mayor apoyo financiero mediante la iniciativa Action Plan for Resilience in Crisis Prone Countries ${ }^{33}$.

Las autoridades libanesas utilizaron este contexto favorable para incrementar su presión sobre la UE con vistas a lograr más recursos, lo que fue negociado en diciembre de 2014 en una Conferencia Internacional cele-

${ }^{29}$ Christina Boswell, «Addressing the Causes of Migratory and Refugee Movements: The Role of the European Union», UNHCR Working Paper, n. ${ }^{\circ} 73$ (2002): 19-21, acceso el 2 de marzo de 2020, https://www.unhcr.org/3e19ac624.pdf

30 Bulcsú Hunyadi y Csaba Molnár, «Central Europe's Faceless Strangers: The Rise of Xenophobia», The Freedom House, 2016, acceso el 8 de octubre de 2019, https:// freedomhouse.org/sites/default/files/July12016_xenophobia_final_brief_FH.pdf

31 Henriette Ruhrmann, y David FitzGerald, «The Externalization of Europe's Borders in the Refugee Crisis, 2015-2016», CCIS WP , 3-8, acceso el 20 de abril de 2020, https://ccis. ucsd.edu/_files/wp194.pdf

32 Council of the European Union, «Conclusions on the European Commission's Communication on Maximising the Development Impact of Migration», 12415/13, Bruselas, 19 de julio de 2013.

33 European Commission, «Action Plan for Resilience in Crisis Prone Countries (20132020)», Commission Staff Working Document, Bruselas, 19 de junio de 2013, SWD 2013, 227 final. 
brada en Berlín bajo el liderazgo del ministro de Exteriores alemán FranckWalter Steinmeier, que aprobó una declaración de apoyo y un sustancial incremento de los recursos para la asistencia humanitaria hacia los refugiados, su integración provisional en los países de acogida, el fortalecimiento de las capacidades administrativas de estos y la mejora de las condiciones de vida de sus habitantes más desfavorecidos. Por el contrario, apenas hubo referencias a la posibilidad de reasentamiento de dichos refugiados en otros lugares mejor preparados para ello, lo que coincidía con las reticencias mostradas por diferentes países de la UE. Al propio tiempo, se subrayaba que la posibilidad de una integración definitiva de esos refugiados en los países de acogida era un derecho soberano de éstos, dando así respuesta a las preocupaciones libanesas ${ }^{34}$. Basándose en ese acuerdo global, las autoridades de Beirut lanzaron a continuación el Plan de Respuesta a la Crisis en Líbano (PRCL) en estrecha colaboración con la ONU y sus agencias, pero buena parte de la financiación corrió a cargo de la UE y de sus integrantes, creciendo desde ese momento el volumen de ayuda al Líbano de forma muy significativa.

La Conferencia de Londres de febrero de 2016 fue escenario de la firma de un Convenio anexo a las Prioridades del Partenariado UE/Líbano, con el objetivo fundamental de mejorar las condiciones de vida de los refugiados sirios en aquel país y de sus comunidades de acogida vulnerables, añadiendo a ello el propósito de su estabilización. Los incentivos financieros proporcionados fueron significativos, estableciéndose en un mínimo de 400 millones de euros para el período 2016-17. Por ejemplo, el apartado de educación se elevó a 59 millones de euros en 2016 destinados a apoyar el programa «Alcanzando a todos los niños con la educación», lanzado por el Gobierno libanés para el período 2016-21 y que tiene por propósito el lograr la escolarización de los niños refugiados (incluyendo a los palestinos), al tiempo que se mejora la calidad educativa en aquellas áreas donde esos refugiados son más abundantes ${ }^{35}$. También se aportaron importantes sumas para la gestión de residuos y basuras (53 millones) y para el sector de la sanidad (11 millones). No podemos olvidar que el acceso a la sanidad en el Líbano se halla todavía muy restringido debido a sus elevados costes, que

34 Así: «Participants noted that any form of integration of refugees from Syria remains a sovereign decision of host and receiving countries». Auswärtiges Amt, «Berlin Communique of the Conference on the Syrian Refugee Situation», 28 de octubre de 2014, acceso el 10 de enero de 2020, https://www.auswaertiges-amt.de/en/newsroom/news/141028-berlinererklaerung/266444

35 EU-Lebanon Association Council, «Decision n. ${ }^{\circ}$ 1/2016 agreeing on EU-Lebanon Partnership Priorities», EU-RL 3001/16, 11 de noviembre de 2016, acceso 2 de noviembre de 2019, https://www.consilium.europa.eu/en/press/press-releases/2016/11/15/eu-lebanonpartnership/ 
se han encarecido con la llegada de refugiados sirios, al tiempo que el sistema sigue sometido al clientelismo político y social ${ }^{36}$.

Estos compromisos fueron confirmados y extendidos en el tiempo a 2018 en la Conferencia de Bruselas de abril de 2017 de apoyo a Siria y sus vecinos $^{37}$. De hecho, la UE se ha esforzado aún más por aportar incentivos para que los refugiados se queden en los países de acogida, poniendo el acento en contrarrestar la tradicional crítica de que la ayuda internacional hace que los refugiados tengan más beneficios que las poblaciones de acogida. Esto ha supuesto el concentrar los recursos de la UE para acción humanitaria en la partida «Forced displacement».

En estas conferencias, las autoridades libanesas subrayaron cómo garantizar la estabilidad política del país a través de una ayuda exterior que no se concentrara solamente en una respuesta de urgencia a la situación creada, sino también en prever las futuras alternativas que se podían ofrecer para asegurar el crecimiento económico y un bienestar generalizado ${ }^{38}$. Este punto de vista fue aceptado por la UE, lo que quedó reflejado en el Marco de Apoyo para el período 2017-20, que promovía el desarrollo en aquellas áreas del Líbano que llevaban más tiempo albergando a refugiados sirios y en las que los recursos se habían resentido, al igual que el empleo ${ }^{39}$. Con estos objetivos, la UE lanzó el proyecto «Strengthening the long-term resilience of subnational authorities in countries affected by the Syrian and Iraqi crises» para el trienio 2019-2021, el cual dedica al Líbano cerca de 15 millones de euros. Su objetivo es reforzar las capacidades de las administraciones a nivel local, de tal manera que permitan una mejora de las condiciones de vida tanto de los refugiados sirios como de la población libanesa allí residente. Dentro del mismo se incluye el reforzamiento de mecanismos para rebajar las tensiones entre ambos colectivos merced a un creciente desarrollo económico que beneficie a todas las partes ${ }^{40}$. En total, el volumen

${ }^{36}$ European External Action Service, «Strengthening the long-term resilience of subnational authorities in countries affected by the Syrian and Iraqi crises», acceso el 6 de noviembre de 2019, http://eueuropaeeas.fpfis.slb.ec.europa.eu:8084/delegations/ lebanon/75493/strengthening-long-termresilience-subnational-authorities-countries-affectedsyrian-and-iraqi_en

37 Brussels Conference I, «Annex: Supporting resilience and development effort of host countries and refugees in the context of the Syrian crisis», Bruselas, 4-5 de abril de 2017.

38 Republic of Lebanon, «Lebanon Statement of Intent». Conferencia de Londres, 4 de febrero de 2016, acceso el 22 de marzo de 2020, https://assets.publishing.service.gov.uk/ government/uploads/system/uploads/attachment_data/file/498026/Supporting_Syria_the_ Region_London_2016_-_Lebanon_Statement.pdf

39 European Commission, «Programming...», 3.

40 European External Action Service, «Strengthening the long-term resilience of subnational authorities in countries affected by the Syrian and Iraqi crises», 2019, acceso el 6 de noviembre de 2019, http://eueuropaeeas.fpfis.slb.ec.europa.eu:8084/delegations/ 
de ayuda desembolsado por la UE hacia los refugiados sirios en el Líbano y sus comunidades de acogida, así como la correspondiente a cooperación bilateral, alcanzó la cifra de 1.400 millones de euros desde 2011. Sin embargo, los avances logrados en los objetivos previstos por la UE han sido bastante escasos ${ }^{41}$.

\section{Entre la europeización y el statu quo}

La política de la UE hacia el Líbano pretendía inicialmente extender hacia ese país algunos de sus propios valores como la democracia, el respeto a los derechos humanos y una gobernanza eficaz, dentro de una estrategia de europeización ${ }^{42}$. Sin embargo, los resultados en esos terrenos son muy limitados. Así, las élites políticas libanesas tienen muy pocos incentivos para cambiar una situación que les mantiene en una posición de liderazgo sobre sus respectivas comunidades mediante el control clientelista de las actividades económicas y de las políticas sociales. Si estas últimas dejaran de responder a criterios clientelistas, entonces su propio poder social quedaría afectado. Así que la respuesta gubernamental ha consistido más bien en preservar el statu quo y utilizar los nuevos recursos para paliar algo la crisis humanitaria entre los refugiados, así como para fortalecer su tradicional dominio comunitario. Y la política europea no ha hecho un gran esfuerzo por cambiar esto, prefiriendo adaptarse al contexto libanés con el objetivo de garantizar la estabilidad ${ }^{43}$.

Así, dado el elevado nivel de corrupción en la Administración libanesa, es habitual que los refugiados deban pagar ciertas sumas de dinero a intermediarios o funcionarios locales simplemente para aparecer registrados como tales y poder beneficiarse de las ayudas establecidas. Por otra parte, la distribución de los recursos no se ha hecho precisamente atendiendo al

lebanon/75493/strengthening-long-termresilience-subnational-authorities-countries-affectedsyrian-and-iraqi_en

${ }^{41}$ Morgan Meaker, «When Aid Funds a Country-Not Its Refugees», Devex, 10 de marzo de 2017, acceso el 7 de febrero de 2029, https://www.devex.com/news/when-aid-funds-acountry-not-its-refugees-89744

${ }^{42}$ Tanja Börzel define europeización como «a process by which domestic policy areas become increasingly subject to European policy-making», pudiendo afectar a países de la UE, pero también a sus vecinos. Tanja Börzel, «Towards Convergence in Europe? Institutional Adaptation to Europeanization in Germany and Spain». Journal of Common Market Studies, n. ${ }^{\circ}$ 39, 4 (1999): 573-96.

43 Ana Uzelac y Jos Meester, «Is there protection in the region? Leveraging funds and political capital in Lebanon's refugee crisis». Clingendael Institute, CRU Report, La Haya, julio 2018, acceso el 18 de marzo de 2020, https://www.clingendael.org/publication/ leveraging-funds-political-capital-lebanons-refugee-crisis 
criterio de mayor necesidad, sino que, como suele ser habitual en el Líbano, el reparto se ha basado en un equilibrio confesional que garantiza el mantenimiento del modelo clientelista existente ${ }^{44}$.

Por último, la doble crisis económica y política que vive el país desde 2019 está afectando negativamente a la visión que muchos libaneses tienen de los refugiados. El muy elevado volumen de deuda pública (cuyo pago resulta complejo) y la consiguiente caída de la credibilidad de los políticos generan un entorno en el que crece el sentimiento según el cual los refugiados constituyen un lastre para el desarrollo del país ${ }^{45}$. El ambiente social existente entre libaneses y refugiados sirios no ha hecho más que deteriorarse, algo en lo que el papel jugado por la clase política (y por líderes sociales) ha sido fundamental, ya que ha adoptado un discurso en el que los refugiados son identificados como una amenaza económica, identitaria y violenta. En el primer caso, han proliferado las acusaciones que vinculaban a los refugiados con el empobrecimiento de los libaneses, con el encarecimiento del coste de la vida, con el empeoramiento en la calidad de los servicios públicos y con el alto desempleo. Por ejemplo, el Patriarca maronita Bishara al-Rai acusó a los refugiados de «quitar a los libaneses el pan de sus bocas, haciéndolos caer en la pobreza y la privación», por lo que exigió su inmediata repatriación ${ }^{46}$. Por su parte, en junio de 2019, el Movimiento Patriótico Libre lanzó una campaña pública contra las empresas que dan trabajo a ciudadanos sirios en la que voluntarios de la sección juvenil del partido se desplegaron por las calles denunciando a estas empresas y repartiendo octavillas en las que se señalaba «Proteged a los trabajadores libaneses y denunciad a los infractores. Siria es un lugar seguro para el retorno y el Líbano ya no puede aguantar» ${ }^{47}$.

El entonces Primer Ministro Saad Hariri se encontraba en una posición difícil en este tema, puesto que debía conciliar su imagen de defensor de la comunidad suní con la de líder del Líbano, buena parte de cuyos habitantes es suspicaz ante la llegada masiva de refugiados suníes. De ahí que Ha-

${ }^{44}$ Meaker, «When Aid...»

45 Mohammad A. Ibrahim y Ammar Hamou, A., «As the Nation Defaults, Lebanon Turns Its Back on Syrian Refugees», Syria Direct, 10 de Marzo de 2020, acceso 20 de marzo de 2020, https://syriadirect.org/news/as-the-nation-defaults-lebanon-turns-its-back-on-syrianrefugees/

46 Ellen Francis, «Hostility Grows toward Syrian Refugees in Lebanon», Reuters, 28 de Agosto de 2020, acceso el 6 de febrero de 2020, https://www.reuters.com/article/us-lebanonrefugees-tension-idUSKCN1B8128

47 Kareem Chehayeb, «Anti-Syrian Refugee Sentiment Ramps Up in Increasingly Hostile Lebanon», Middle East Eye, 14 de junio de 2019, acceso el 9 de febrero de 2020, https://www.middleeasteye.net/news/anti-syrian-refugee-sentiment-ramps-increasinglyunwelcome-lebanon 
riri haya por un lado sostenido el derecho de asistencia humanitaria para los refugiados, mezclado con una apelación a la comunidad internacional para que facilite su repatriación y con una demanda de creciente ayuda económica no sólo para cubrir sus necesidades, sino también para mejorar la vida de los libaneses. Sin embargo, Hariri es partidario de no forzar a nadie a retornar a Siria, algo que suele subrayar en sus contactos con la UE. Tampoco es sencilla la posición de los partidos chiíes Hezbollah y Amal, ya que si bien los refugiados constituyen un grupo que mayoritariamente no simpatiza con ellos por el apoyo que prestan al régimen de Damasco, al propio tiempo su retórica panarabista y panislámica no encajaría bien con su rechazo a acogerlos, por lo que siempre han mantenido un discurso de aceptación ${ }^{48}$.

En lo relativo a la amenaza de la violencia, a menudo los refugiados han sido vinculados en el discurso político tanto con la delincuencia común como con el yihadismo, de tal modo que algunas medidas hacia ellos han sido justificadas como necesarias para prevenir la expansión de esos fenómenos. En ese terreno, tanto el Presidente Aoun como Hezbollah se han presentado como los garantes del carácter pluralista del Líbano frente a las amenazas de grupos como Jabhat al-Nusra o el Estado Islámico. Los enfrentamientos de Arsal o Trípoli han sido puestos como ejemplo de esa lucha antiyihadista, que ha implicado a menudo acciones poco respetuosas con los derechos humanos. Pero ello ha contribuido a fortalecer la imagen del Movimiento Patriótico Libre y de Hezbollah ante un electorado atemorizado por el peligro de expansión yihadista ${ }^{49}$.

Por último, la amenaza identitaria se debe a que algunas comunidades (especialmente los cristianos, pero también los chiíes) perciben en los refugiados sirios un factor desestabilizador entre los distintos grupos confesionales, de manera que su asentamiento permanente e incluso su naturalización podrían implicar un cambio en las características sociales y en los equilibrios de poder, modificando lo que ellos conciben como identidad libanesa. Así, el falangista Sami Gemayel consideró que la presencia de los refugiados constituía una amenaza que debía ser afrontada con la creación de un nuevo gobierno de salvación por constituir una «amenaza exis-

48 Georgi Azar, «Hariri Calls on EU to Exude Pressure on Syria to Return Refugees», An Nahar, 14 de marzo de 2019, acceso el 4 de enero de 2020, https://en.annahar.com/ article/948326-hariri-calls-on-eu-to-exude-pressure-on-syria-to-return-refugees. Alexander Betts, Ali Ali y Fulya Memisoglu, «Local politics and the Syrian Refugee Crisis. Exploring Responses in Turkey, Lebanon and Jordan». University of Oxford: Refugee Studies Centre,17, acceso el 20 de enero de 2020, https://www.refugee-economies.org/assets/ downloads/Local-politics-of-syrian-refugee-crisis_report-web.pdf $17-18$.

49 International Crisis Group, «Arsal...», 12. Betts, Ali y Memisoglu, «Local politics...», 
tencial», además de recalcar el peligro derivado de que entre esos refugiados hubiera un número importante de palestinos. Por su parte, el Presidente Aoun ha afirmado que la naturalización de los refugiados en el futuro constituiría una vulneración de la Constitución libanesa ${ }^{50}$.

En los últimos años ha habido una política más activa por parte de las autoridades de Beirut a la hora de «convencer» a los refugiados de que deben retornar a su país. Por un lado, muchos de ellos carecen de acceso a la escolarización y los cortes de suministro eléctrico son constantes, al tiempo que tienen serios obstáculos para poder trabajar. Sus viviendas siguen siendo muy precarias, mientras se lleva a cabo la demolición de aquellas estructuras de cemento que los cobijan, las cuales son consideradas ilegales. Según las autoridades libanesas, la política de retorno «voluntario» ha permitido que 130.000 sirios hayan vuelto a su país entre diciembre de 2017 y marzo de 2019. Es cierto que al mismo tiempo se ha dado una cierta movilización social contra esto, denunciando por ejemplo el carácter racista y populista de la campaña del MPL, pero lo cierto es que el clima de estigmatización de los refugiados se ha extendido a buena parte del país ${ }^{51}$.

El debate sobre los refugiados se ha mezclado en los últimos meses con la cuestión de si el Líbano debe restablecer o no sus relaciones con Siria, reconociendo así a Bashar al-Asad como líder de su país. Los partidos del 8 de Marzo sostienen que si se quiere convencer a las autoridades de Damasco de que creen las condiciones propicias para ese retorno, también sería necesario establecer un diálogo político con ellas, lo que por otra parte implicaría reconocer su victoria militar. Ya en el verano de 2017 un acuerdo entre la Seguridad General ${ }^{52}$, las autoridades sirias y la organización yihadista Jabhat al-Nusra permitió la evacuación pacífica de numerosos combatientes y de refugiados afines desde la ciudad libanesa de Arsal hacia el noroeste de Siria. Para el líder de Hezbollah, Hassan Nasrallah, esto suponía una muestra de cómo el diálogo político entre ambos Estados

50 «Gemayel Sounds Alarm about Refugee Crisis», Now, 15 de abril de 2013, acceso el 27 de marzo de 2020, http://now.mmedia.me/lb/en/archive/gemayel-sounds-alarmabout-refugee-crisis. «Gemayel Calls on EU to Work on Refugess` Return with Key Regional Players», Kataeb Party, acceso el 9 de febrero de 2020, https://www.kataeb. org/local/2019/04/10/gemayel-calls-on-eu-to-work-on-refugees-return. Maja Janmyr, M., «Why Lebanon Objects to Calls for Voluntary Return of Syrians?», Syria Deeply, 4 de mayo de 2018, acceso el 3 de febrero de 2020, https://www.newsdeeply.com/syria/ community/2018/05/04/why-lebanon-objects-to-calls-for-voluntary-return-of-syrians-2

${ }^{51}$ Rebecca Collard, «Syrian Refugees Return from Lebanon to an Uncertain Future», Financial Times, 4 de julio de 2018, acceso el 10 de junio de 2020, https://www.ft.com/ content/797e5f14-7af3-11e8-8e67-1e1a0846c475. Chehayeb, «Anti-Syrian...»

${ }^{52} \mathrm{La}$ Seguridad General es la fuerza policial encargada de las fronteras libanesas y de los ciudadanos extranjeros. 
podía reducir el peso de la presencia de refugiados, por lo que debía ser el camino a seguir ${ }^{53}$. También el Presidente del Parlamento (y líder de Amal) Nabih Berri subrayó que «We can no longer bury our heads in the sand. We must make agreements with the [Syrian] state to return refugees» ${ }^{54}$. Una buena ocasión para promover este debate fue la celebración en Beirut de la Cumbre Económica de la Liga Árabe (20 enero de 2019), en la que el Presidente Aoun estudió la idea de invitar a las autoridades sirias como primer paso para su retorno a las instituciones internacionales. Sin embargo, Washington bloqueó esta iniciativa, amenazando a Aoun con sanciones en el caso de que fuera adoptada ${ }^{55}$.

Por su parte, los partidos del 14 de Marzo rechazan esa normalización de relaciones, pero al propio tiempo exigen de Damasco el que facilite las repatriaciones, incluso sin que se haya llegado a un pacto político que ponga fin a la guerra. Esto no deja de ser complejo, dado que difícilmente el Líbano podría convencer a Siria de esta necesidad si al propio tiempo elude un diálogo político con Asad. La solución propuesta radicaría en que fueran otros actores (Naciones Unidas, la UE y especialmente Rusia) quienes desarrollaran esa labor de interlocución con las autoridades sirias. En este sentido, el Primer Ministro Hariri solicitó a la UE que utilizara su presión para convencer a Siria de que facilitara ese retorno ${ }^{56}$. Las Fuerzas Libanesas han sido el partido del 14 de Marzo que más ha insistido en la necesidad de acelerar el proceso de repatriación, haciendo en marzo de 2019 una propuesta formal en ese sentido que dejaba en manos del Gobierno esa decisión, siendo la Seguridad General la institución que debía manejar la aplicación práctica de las repatriaciones al ser la única institución pública libanesa que mantiene de forma abierta su comunicación con las autoridades sirias. Por su parte, el líder falangista Sami Gemayel en una reunión del Partido Popular Europeo en Bruselas urgió a la UE a trabajar para la repatriación de los refugiados ${ }^{57}$.

53 «Nasrallah Urges Government to Negotiate with Syria on Refugess, Says Keen on Kuwait Ties», Naharnet, 4 de agosto de 2017, acceso el 2 de abril de 2020, http://www. naharnet.com/stories/en/233776

54 «Refugees Leave, Berri Urges Deal with Syria», The Daily Star, 9 de abril de 2019, acceso el 8 de julio de 2020, https://dailystar.com.lb/News/Lebanon-News/2019/Apr09/480736-refugees-leave-berri-urges-deal-with-syria.ashx

55 C. Jacob, «Debate in Lebanon on Inviting Syria to Arab Economic Summit in Beirut, Normalizing Relations with It», MEMRI, 18 de enero de 2019, acceso el 7 de diciembre de 2019, https://www.memri.org/reports/debate-lebanon-inviting-syria-arab-economic-summitbeirut-normalizing-relations-it

56 Georgi Azar, «Hariri Calls...»

57 «Kouyoumjian Details LF Refugee Return Proposal», The Daily Star, 4 de marzo de 2019, acceso el 19 de junio de 2020, https://dailystar.com.lb/News/Lebanon-News/2019/Mar04/477949-kouyoumjian-details-lf-refugee-return-proposal.ashx. «Gemayel Calls...» 
Pero este tema ha provocado claros desencuentros en las relaciones entre la UE y el Líbano, ya que aquella considera que el retorno de los refugiados a Siria es prematuro por no darse todavía unas condiciones de seguridad para ellos y ha exigido como condición previa la firma de un acuerdo político que ponga fin al conflicto armado, tal como expresó la Alta Representante Federica Mogherini en su visita al Líbano en diciembre de 2017. Por su parte, Beirut cree que dichas condiciones sí que existen ya, puesto que amplias zonas del país no registran combates. Así, el líder del MPL Gebran Bassil ha criticado reiteradamente la visión de la UE de que los refugiados deben continuar en el Líbano hasta que se alcance un acuerdo político entre las partes enfrentadas, lo que pondría en riesgo al país al fomentar el asentamiento definitivo de esos refugiados dado que un acuerdo en Siria no parece cercano. También ha expresado la posibilidad de que la presencia de los refugiados provoque una extensión de la guerra civil al Líbano, al tiempo que ha lanzado un aviso a la UE sobre las hipotéticas consecuencias que esto podría tener sobre la llegada de refugiados desde el Líbano hacia Europa. De hecho, esta posición del MPL llevó a este partido a organizar una manifestación en Bruselas para expresar su protesta por las políticas de la UE en ese tema ${ }^{58}$. Ante estos desencuentros, el Líbano confía cada vez más en Rusia para que medie ante Asad con vistas a solventar este problema.

Finalmente, la condicionalidad de las ayudas de la UE ha perdido su efecto ante la creciente coerción ejercida sobre los refugiados para forzar su repatriación a Siria, así como ante los abundantes casos de vulneración de los derechos humanos por parte de los cuerpos de seguridad libaneses bajo el paraguas de la lucha antiterrorista, por lo que el avance en la europeización resulta muy cuestionable ${ }^{59}$.

Sin embargo, la profunda crisis en la que se encuentra el país desde el otoño de 2019 y que condujo a una fuerte oleada de protestas que reclamaban la retirada de la clase política en su conjunto y la creación de un Gobierno tecnocrático ha abierto una nueva vía para la intervención europea.

58 Bassil fue muy explícito al señalar los riesgos para la UE: «Mon avertissement aujourd'hui est le suivant: des centaines de milliers de Libanais, de Syriens et de Palestiniens pourraient quitter le Liban et se rendre chez vous, en raison de ce qui se trame contre nous». «Bassil lors du Forum Mondial sur les Refugées: 'C'est qui s'est passé en Syrie risque de se produire au Liban`», L'Orient-le-Jour, 17 de diciembre de 2019, acceso el 24 de junio de 2020, https://www.lorientlejour.com/article/1198989/bassil-lors-du-forum-mondial-sur-lesrefugies-ce-qui-sest-passe-en-syrie-risque-de-se-produire-au-liban.html

59 Sara Stachelhaus, «Stabilization Via Change? The European Union`s Support for Human Rights Advocacy in Lebanon», Beirut, Heinrich Böll Stiftung, 2019, 5, acceso el 18 de marzo de 2020, https://lb.boell.org/en/2019/04/16/stabilization-change-european-unionssupport-human-rights-advocacy-lebanon-0 
La caída del Primer Ministro Saad Hariri en octubre de ese año no solucionó los acuciantes problemas presupuestarios y el enorme peso de la deuda pública. Pero la situación fue aún más acuciante con el accidente en un depósito de explosivos el 4 de agosto de 2020, que provocó graves destrozos y un gran volumen de víctimas (por encima de doscientas) en la zona portuaria de Beirut, lo que animó más las protestas y forzó la caía del Gobierno de Hassan Diab. La reacción europea fue protagonizada por el Presidente francés, Emmanuel Macron, que ha intentado mediar en la crisis y facilitar la creación de un Gobierno reformista, al tiempo que ha subrayado la idea de reforzar la condicionalidad de la ayuda de la UE. Sin embargo, el retorno de Saad Hariri al frente del mismo más bien parece indicar que el sistema político es muy resistente al cambio y que la capacidad de la UE de proyectar su agenda de reforma resulta bastante limitada por su propio interés en preservar la estabilidad del país con el objetivo de evitar una oleada de refugiados hacia su propio territorio. El problema es que la falta de cambios puede igualmente propiciar un éxodo poblacional, pero en este caso de nacionalidad libanesa, ante un futuro cada vez más sombrío para el país ${ }^{60}$.

\section{Conclusiones}

La política de la UE hacia los refugiados sirios en el Líbano se ha centrado fundamentalmente en lograr la permanencia de estos en el país, si bien hay que distinguir una cierta evolución en la misma, ya que inicialmente trató de promover el que las autoridades de Beirut cumplieran de forma más estricta el principio de no repatriación forzosa, al tiempo que los gobiernos europeos rechazaban la posibilidad de reasentamiento de algunos refugiados en su territorio. Sin embargo, en los últimos años la UE ha sido menos estricta en lo relativo a salvaguardar dicho principio y en el avance del respeto a los derechos humanos, de modo que la condicionalidad en la ayuda ha perdido eficacia por cuanto han prevalecido los objetivos de estabilidad y de externalización del problema de los refugiados en la UE. En otras palabras, la capacidad de la UE como potencia normativa es cada vez menor debido a su dependencia con respecto a la colaboración libanesa para controlar los flujos de personas hacia Europa.

A partir de 2012 existía en el Líbano un riesgo cierto de que algunos refugiados pudieran convertirse en refugiados-soldado que utilizaran el

${ }^{60}$ International Crisis Group, «How Europe Can Help Lebanon Overcome Its Economic implosion», Middle East Report, n. ${ }^{\circ}$ 259, 30 de octubre de 2020, 7-8, acceso el 2 de noviembre de 2020, https://d2071 andvip0wj.cloudfront.net/219-lebanon-economicimplosion.pdf 
territorio del país para continuar su enfrentamiento contra el régimen de Damasco. En ello influían especialmente factores como su cercanía a la frontera y la presencia de grupos yihadistas libaneses con los que podían cooperar en esa lucha e incluso involucrarse en la propia política libanesa. La acción europea no tuvo en cuenta esto al insistir en la permanencia de los refugiados en el país. Sin embargo, tampoco se puede sobreestimar ese peligro, ya que muy pocos refugiados se implicaron en esas actividades. En otras palabras, las autoridades libanesas exageraron la amenaza con vistas a extraer el máximo de recursos europeos. Igualmente, utilizaban esa amenaza yihadista para reforzar su posición interna.

La acción de la UE ha mostrado una eficacia bastante limitada a la hora de contribuir a la mejora de las condiciones de vida tanto de los refugiados sirios y palestinos como de los ciudadanos libaneses que viven en la pobreza. Es cierto que esas ayudas les han permitido lograr alimentos y servicios básicos, pero la falta de reformas en el Líbano y el mantenimiento de un sistema de protección social basado en criterios clientelistas ha provocado un deterioro en las condiciones de vida de muchos libaneses pobres, lo que queda reflejado en las protestas que tienen lugar en el país desde finales de 2019 y que muestran un creciente hartazgo social. Por su parte, si la UE intentaba fortalecer la posición interna de la Coalición 14 de Marzo, aquí el fracaso es evidente, ya que en las elecciones de mayo de 2018 ésta perdió posiciones, mientras que su líder Saad Hariri tuvo que dimitir como Primer Ministro en octubre de 2019 a raíz de esas protestas.

Hasta qué punto la UE ha contribuido a la estabilización del Líbano es un tema controvertido. No hay duda de que el país ha sido capaz de mantener (a duras penas) la paz interior en unas condiciones que parecían propicias para la extensión de los conflictos vecinos, especialmente debido a su debilidad histórica ante las tensiones regionales. Pero también hay que subrayar que se han producido numerosos enfrentamientos armados entre distintas facciones y atentados que han causado cientos de víctimas, dentro de un clima cercano al estallido de una nueva guerra civil.

Más éxito ha tenido la UE a la hora de evitar que los refugiados sirios buscaran su reasentamiento en su territorio. Es verdad que en los años 2014-2016 la presión de la llegada de refugiados fue notable, pero en todo caso resultó bastante menor que en el Líbano, Turquía y Jordania. No obstante, el precio pagado por este logro fue, como se ha dicho antes, el mantener a esos refugiados en unas condiciones de vida muy precarias, al tiempo que su amplia presencia en el Líbano se convertía en un factor de desestabilización interna y de confrontación política en ese país. Igualmente, esta actitud sirvió para que la política de la UE careciera de credibilidad como potencia normativa, ya que promovía unos principios que ella misma no aplicaba. 
La alternativa para la UE hubiera sido asumir directamente un mayor peso en la gestión del problema de los refugiados, descargando parcialmente al Líbano de una tarea para la que el país no estaba preparado y evitando que las tensiones provocaran allí mayor inestabilidad. Esto le habría concedido una mayor credibilidad entre los dirigentes libaneses al ser capaz de aplicar con mayor seriedad unos requisitos de condicionalidad a la ayuda prestada, pudiendo contribuir así a que se comprometieran con un programa reformista que el país necesita desesperadamente ${ }^{61}$. En definitiva, la política europea hacia el Líbano y los refugiados sufrió de una clara falta de coherencia en sus objetivos, inclinándose al final por primar la seguridad interior (mantener los refugiados fuera), aunque esto pudiera conducir a aumentar la inestabilidad en un país vecino.

Estos objetivos contradictorios entre la PESC y el ELSJ ponen en evidencia una vez más cómo el poder económico de la UE no es suficiente en sí mismo para garantizarle un peso adecuado a escala internacional en ausencia de una política coherente en todas sus dimensiones. También demuestra la escasa eficacia de la condicionalidad con países que no son candidatos a ingresar en la UE.

\section{Sobre el autor}

Javier Lion Bustillo es actualmente Profesor Ayudante Doctor en el Departamento de Historia, Teorías y Geografía Políticas de la Universidad Complutense de Madrid. Anteriormente, ha sido Profesor Doctor Asociado en el Departamento de Relaciones Internacionales de la Universidad Pontificia de Comillas /ICADE. Es Doctor en Historia por la Universidad de Cádiz y posee un Master en Política y Gobierno en la Unión Europea por la London School of Economics. Investigador visitante en la Universidad del Sarre (Alemania) y en la London School of Economics. Profesor visitante en el Departamento de Relaciones Internacionales de la Universidad de Coimbra (Portugal). Sus investigaciones se centran en las áreas de: Historia de las Relaciones Internacionales, integración europea, Política Mediterránea de la UE y estudios de paz y conflictos. Ha escrito artículos para publicaciones como Historia y Política, Revista de Estudios Políticos y Revista Española de Ciencia Política. También es autor del libro La Comunidad Europea y la unificación alemana.

61 Uzelac y Meester, «Is There...», 62. 


\begin{abstract}
About the author
Javier Lion Bustillo is currently Lecturer at the Department of Political History, Theories and Geography (University Complutense of Madrid). Previously, he was Part-time Lecturer in International Relations at the Pontifical University of Comillas/ICADE (Madrid, Spain). He holds a $\mathrm{PhD}$ in History from the University of Cádiz (Spain) and a MA in Politics and Government in the EU from the London School of Economics. Visiting researcher at Saarland University (Germany) and the London School of Economics. Visiting professor at the Department of International Relations (University of Coimbra, Portugal). His research is focused on the fields of History of International Relations, European integration, EU Mediterranean Policy, peace and conflict studies. He has written articles for publications like Historia y Política, Revista de Estudios Políticos and Revista Española de Ciencia Politica. He is also the author of the book La Comunidad Europea y la unificación alemana.
\end{abstract}




\section{Derechos de autor}

Los derechos de autor (para la distribución, comunicación pública, reproducción e inclusión en bases de datos de indexación y repositorios institucionales) de esta publicación (Cuadernos Europeos de Deusto, CED) pertenecen a la editorial Universidad de Deusto. El acceso al contenido digital de cualquier número de Cuadernos Europeos de Deusto es gratuito inmediatamente después de su publicación. Los trabajos podrán leerse, descargarse, copiar y difundir en cualquier medio sin fines comerciales y según lo previsto por la ley; sin la previa autorización de la Editorial (Universidad de Deusto) o el autor. Así mismo, los trabajos editados en CED pueden ser publicados con posterioridad en otros medios o revistas, siempre que el autor indique con claridad y en la primera nota a pie de página que el trabajo se publicó por primera vez en CED, con indicación del número, año, páginas y DOI (si procede). Cualquier otro uso de su contenido en cualquier medio o formato, ahora conocido o desarrollado en el futuro, requiere el permiso previo por escrito del titular de los derechos de autor.

\section{Copyright}

Copyright (for distribution, public communication, reproduction and inclusion in indexation databases and institutional repositories) of this publication (Cuadernos Europeos de Deusto, CED) belongs to the publisher University of Deusto. Access to the digital content of any Issue of Cuadernos Europeos de Deusto is free upon its publication. The content can be read, downloaded, copied, and distributed freely in any medium only for non-commercial purposes and in accordance with any applicable copyright legislation, without prior permission from the copyright holder (University of Deusto) or the author. Thus, the content of CED can be subsequently published in other media or journals, as long as the author clearly indicates in the first footnote that the work was published in CED for the first time, indicating the Issue number, year, pages, and DOI (if applicable). Any other use of its content in any medium or format, now known or developed in the future, requires prior written permission of the copyright holder. 